\title{
RESEARCH \\ Optimization of a Molecular Genetic Assay for the Sensitive Detection of KRAS Mutations in Colorectal Carcinoma
}

\author{
Isaac Miao', Sherry A.M. Taylor PhD² \\ 'Class of 2014, Faculty of Medicine, Dalhousie University \\ ${ }^{2}$ Division of Molecular Genetics, Department of Laboratory Medicine, Saint John Regional Hospital, Horizon \\ Health Network and Department of Pathology, Dalhousie University
}

\begin{abstract}
Activating mutations of codons 12 and 13 of the KRAS gene occur frequently in colorectal carcinomas. Clinical trials have shown patients with metastatic colorectal cancer benefit from treatment with tyrosine kinase inhibitors, eg. Vectibix (panitumumab), used to block EGFR overexpression if point mutations in codons 12 or 13 of the KRAS gene are absent. The DxS KRAS mutation kit, mandated by Health Canada as a companion diagnostic, doubled in price to $\$ 200-\$ 500$ per patient tested, making it less attractive for use by clinical laboratories. Using 25 cases of colorectal cancer previously analyzed by an external laboratory, we validated and compared DxS (Amplification Refractory) and a less costly method, SNaPshot (Primer Extension), for KRAS mutation analysis as well as two extraction methods, Qiagen FFPE and PicoPure. We demonstrated that DNA purified using the Qiagen FFPE kit produced more consistent results. KRAS mutation status was obtained for all samples assayed and were compared with results from the external laboratory. Both DxS and SNaPshot met the required analytic sensitivity of $1 \%$ mutant to wild-type background. One sample was discordant by both assays with those previously reported due to either the assays or sampling from a different location in the tumour tissue. SNaPshot detected a Gly13Asp variant as an artefact in addition to the correct genotype in four samples. DNA quality from the fixed tissues and PCR amplification conditions were investigated as the cause. After further validation and refinement of the amplification conditions, the SNaPshot method has now been adopted for clinical use.
\end{abstract}

In Canada, colorectal cancer (CRC) is the second leading cause of cancer death in men and women, with an estimated 9,200 deaths in 2012 (12\% of all cancer deaths) and 23,300 estimated new cases annually. ${ }^{1}$ Epidermal growth factor receptor (EGFR) overexpression has been found to lead to uncontrolled growth, proliferation, and differentiation of tumour cells, and is often found to be activated in colon cancer. For this reason, anti-EGFR therapies such as Erbitux (cetuximab) and Vectibix (panitumumab) have been developed.

However, using immunostaining techniques, presence of EGFR proteins has not been shown to correlate with therapy responsiveness. ${ }^{2}$ Evidence of CRC patients with EGFR-negative tumours responding to cetuximab treatment has led researchers to find the Kirsten rat viral oncogene (KRAS) as a more suitable biomarker. In fact, several studies have demonstrated high response rates to cetuximab and panitumumab in patients with wild-type KRAS genes, whereas those with mutant versions did not benefit from the treatment and instead incurred increased side effects. ${ }^{3}$

KRAS, a GTPase protein, is known to be involved in signal transduction pathways involving growth factors and to play a large role in CRC. Its mutation status has been found to be a very good predictor of panitumumab therapy outcome, as it is thought that patients with activating mutations in their KRAS gene are continuously producing proteins triggering a signalling cascade independent of EGFR.

While this $\$ 4000$ per treatment monoclonal antibody efficaciously acts on EGFR, about $40 \%$ of patients possess a mutated KRAS gene that is always "turned on" and will demonstrate poor response to this therapy. ${ }^{3}$ The most common KRAS mutations have been found in codons 12 and 13 of exon 2, which has resulted in a wide range of PCR-based technologies being developed in an attempt to detect the mutations in these codons. Health Canada has approved the DxS TheraScreen KRAS testing kit (Qiagen) for the detection of seven common mutations in the KRAS gene and as a companion diagnostic for panitumumab. ${ }^{4}$

The DxS kit advertises a detection capability of $1 \%$ mutant DNA in a wild-type background. However, due to the relatively low number of KRAS mutation analysis requests in the province (50-100 per year), the use of the DxS kit results in each test costing from $\$ 200$ to $\$ 500$ per patient, excluding the extraction process, consumables, 
or work units. We therefore sought to evaluate equally sensitive diagnostic alternatives to the Health Canada approved DxS kit in an attempt to minimize costs and turnaround time. DNA sequencing, while considered to be the gold standard, is known to not be reliably sensitive if the percentage of KRAS mutation positive cells is less than $20 \% .^{5}$ PCR with co-amplification at lower denaturation temperature (COLD-PCR) with high resolution melt analysis and RFLP analysis were also considered but may require confirmatory sequence analysis. ${ }^{6}$ It has been recommended by Canadian, US, and European groups that KRAS testing should be able to detect mutations, if present, in $1-5 \%$ of cells. ${ }^{7-9}$ Therefore the assay selected should be able to detect $1-5 \%$ of KRAS positive cells in tumour samples and identify the mutation present without the need for a second assay. The SNaPshot assay (Life Technologies) can be used to detect and differentiate mutant or polymorphic alleles (single nucleotide polymorphisms or SNPs) which differ at a single nucleotide base. It is a primer extension based method which uses PCR followed by single base extension using an unlabelled primer and fluorescently labelled dideoxynucleotides (ddNTP). The SNaPshot assay is reported to have a sensitivity in the range of the DxS kit, which makes it a reasonable alternative. In this paper, we compare the DxS kit with the SNaPshot assay system for use in determining KRAS mutation status in metastatic colorectal cancer.

\section{Methods}

DNA Specimens:

Formalin-fixed paraffin-embedded (FFPE) blocks of surgical biopsies of 25 colorectal cancer patients were acquired from the Saint John Regional Hospital Archives with Research Ethics Board approval and de-identified by labelling the samples 1-25. As these blocks had been previously sent out to an external laboratory for KRAS mutation analysis, their results were acquired and considered the reference standard. Specimens were between one to eight years old. DNA extracted from FFPE samples as old as 30 years have been demonstrated to be amplifiable. ${ }^{9}$ An experienced pathologist highlighted the location of the tumour on a hematoxylin and eosin stained (H\&E) slide and the area was macro-dissected from an unstained $3 \mu \mathrm{m}$-thick section using a disposable sterile scalpel.

\section{Extractions:}

To deparaffinise the tissue, a section was transferred into a microcentrifuge tube with xylene and centrifuged. The supernatant was then removed and the tissue was washed with anhydrous ethanol and centrifuged once more at high speed. The supernatant was then removed and the tube was left open to incubate at room temperature to evaporate the solvents for 10 minutes. Genomic DNA was isolated with the PicoPure DNA Extraction Kit (Arcturus) according to the manufacturer's instructions.

Tissue extractions were also performed using the Qiagen DNA FFPE Tissue Extraction Kit (Qiagen) and incubated for 16 hours as per the manufacturer's recommendations to completely lyse the sample. Yields of DNA specimens from both extraction methods were quantified using a NanoDrop ND1000 spectrophotometer (NanoDrop Technologies)

\section{PCR of the KRAS gene:}

Exon 2 of the KRAS gene was amplified using DNA specimens from both extraction methods and forward and reverse primers described. ${ }^{6}$ This was done to provide the template for the SNaPshot assays and to control for DNA concentration in the DxS assay. PCR was performed in a $20 \mu \mathrm{L}$ volume containing $11.4 \mu \mathrm{L}$ of PCR buffer, $0.2 \mu \mathrm{L}$ of FastStart Taq DNA Polymerase (Roche) and $1 \mu \mathrm{L}$ of DNA. The PCR reaction was carried out at $94^{\circ} \mathrm{C}$ for 10 minutes followed by 30 cycles of $94^{\circ} \mathrm{C}$ for $30 \mathrm{~s}, 55^{\circ} \mathrm{C}$ for 30 s and $72^{\circ} \mathrm{C}$ for 30 s with a final 10 minute extension step at $72^{\circ} \mathrm{C}$ using a Veriti 96 Well Fast Thermal Cycler (Applied Biosystems).

PCR products were analyzed on a $2 \%(\mathrm{w} / \mathrm{v})$ agarose gel (Bio-Rad) electrophoresis run stained with ethidium bromide $2 \mu \mathrm{l} / \mathrm{ml}$ (Amresco) to assess KRAS amplification and for comparisons between the two extraction methods.

\section{SNaPshot:}

Post-PCR products were cleaned with EXO-SAP and mixed with four primers corresponding to the four mutation sites (codon 12 position 1, codon 12 position 2, codon 13 position 1, and codon 13 position 2) as described in reference. ${ }^{10}$ Fluorescently labelled ddNTPs were added and the multiplex $\mathrm{SNaPshot}$ procedure was carried out according to the manufacturer's instructions on samples from both extraction methods. Samples were analyzed on an ABI 3130 Genetic Analyzer.

$D x S:$

DNA samples from both extraction methods were analyzed using the commercial DxS kit as per manufacturer's instructions. The seven most common KRAS exon 2 mutations (Table 1) were analyzed by means of a proprietary real-time PCR assay. The manufacturer claimed a sensitivity of $1 \%$ mutant in a 
background of wild-type genomic DNA. Samples were analyzed on an ABI 7500 Fast Real Time PCR system.

\section{Cost Analysis:}

A comparison of costs of materials and labour was made between the commercial DxS kit, the SNaPshot method, and sending the sample to an external laboratory.

Table 1. KRAS gene mutations detected by the DxS and SNaPshot assays.

\begin{tabular}{|c|c|c|c|}
\hline Mutation & Base Change & DxS & SNaPshot \\
\hline p.Gly12Ser & $\underline{G} G T>\underline{A} G T$ & $x$ & $x$ \\
\hline p.Gly12Arg & $\underline{G G T}>\underline{G G T}$ & $x$ & $x$ \\
\hline p.Gly12Cys & $\underline{G} G T>\underline{T} G T$ & $x$ & $x$ \\
\hline p.Gly12Asp & $\mathrm{G} \underline{\underline{G} T}>\mathrm{G} \underline{A} T$ & $x$ & $x$ \\
\hline p.Gly12Ala & $G \underline{G} T>G \underline{T} T$ & $x$ & $x$ \\
\hline p.Gly12Val & $\underline{G G T}>\underline{T} G T$ & $x$ & $x$ \\
\hline p.Gly13Ser & $\underline{G G C}>\underline{A G C}$ & & $x$ \\
\hline p.Gly13Arg & $\underline{G G C}, \underline{G} G C$ & & $x$ \\
\hline p.Gly13Cys & $\underline{G G C}>\underline{T} G C$ & & $x$ \\
\hline p.Gly13Asp & $G \underline{G} C>G \underline{A} C$ & $x$ & $x$ \\
\hline p.Gly13Ala & $G \underline{G C}>G \underline{C} C$ & & $x$ \\
\hline p.Gly13Val & $G \underline{G} C, G \underline{I} C$ & & $x$ \\
\hline
\end{tabular}

\section{Results}

\section{Extractions}

While the Qiagen extraction method produced lower yields, requiring multiple transfers of the sample between vials and columns, it consistently produced superior results in DxS runs, as shown in Figure 1. The PicoPure method produced higher yields due to the sample remaining in one vial for the duration of the extraction but yielded less amplification product. This was determined by comparing the number of PCR cycles required until amplification was observed. The Qiagen method consistently exhibited amplification in fewer cycles than the PicoPure process indicating either a greater amount of amplifiable target DNA was present in the extracted samples or alternatively that lower concentrations of inhibitors were present.

Results from the external referral lab, SNaPshot, and DxS runs are detailed in Table 2. The DxS method was found to be highly concordant with the external laboratory (95\%) and was able to detect mutations in

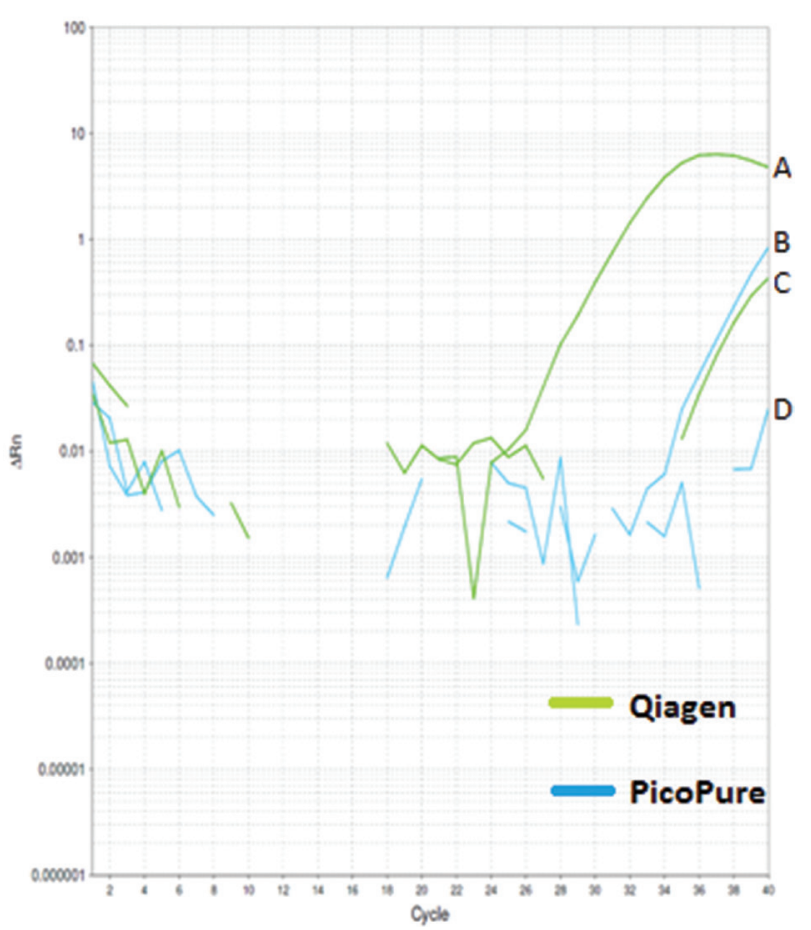

Figure 1. The use of the DxS kit comparing the Qiagen and PicoPure extraction methods from the same tumour DNA sample carrying the $p$.Gly12Ser mutation. The $x$-axis shows the number of elapsed PCR cycles. The $y$-axis represents amount of DNA detected (by fluorescence). The Qiagen control sequence (curve A) has amplified with fewer cycles than the PicoPure control sequence (curve B). The amplification of the p.GLy12Ser mutation using the Qiagen extracted sample also occurred at a lower cycle number (curve $C$ ) than for the PicoPure extracted sample (curve D). For the Picopure extracted sample a false negative mutation result was obtained.

concentrations as low as $0.5 \%$, meeting its advertised sensitivity of $1 \%$. An example of a DxS run of 10 samples (the maximum amount allowed as per manufacturer instructions) can be seen in Figure 2, with varying levels of mutation percentages. The SNP genotyping method, SNaPshot, also resulted in 95\% concordance with the external laboratory. In addition, both methods were able to detect a mutation in sample 19 that the external laboratory could not. A SNaPshot result chart of corresponding samples can be seen in Figure 3. Highlighted results in Table 2 demonstrate discordance in results between SNaPshot/DxS results and the external laboratory.

\section{Cost analysis}

KRAS mutation detection by either the DxS kit or the SNaPshot method showed both to have turnaround times that were less costly than sending out to an 
external laboratory. Combined with the preferred Qiagen extraction method, the SNaPshot method was able to be performed at a lower cost at $\$ 106$ per sample than the DxS Kit at \$266.

\section{Task}

Extraction

Analysis

xternal lab analysis for KRAS

mutations

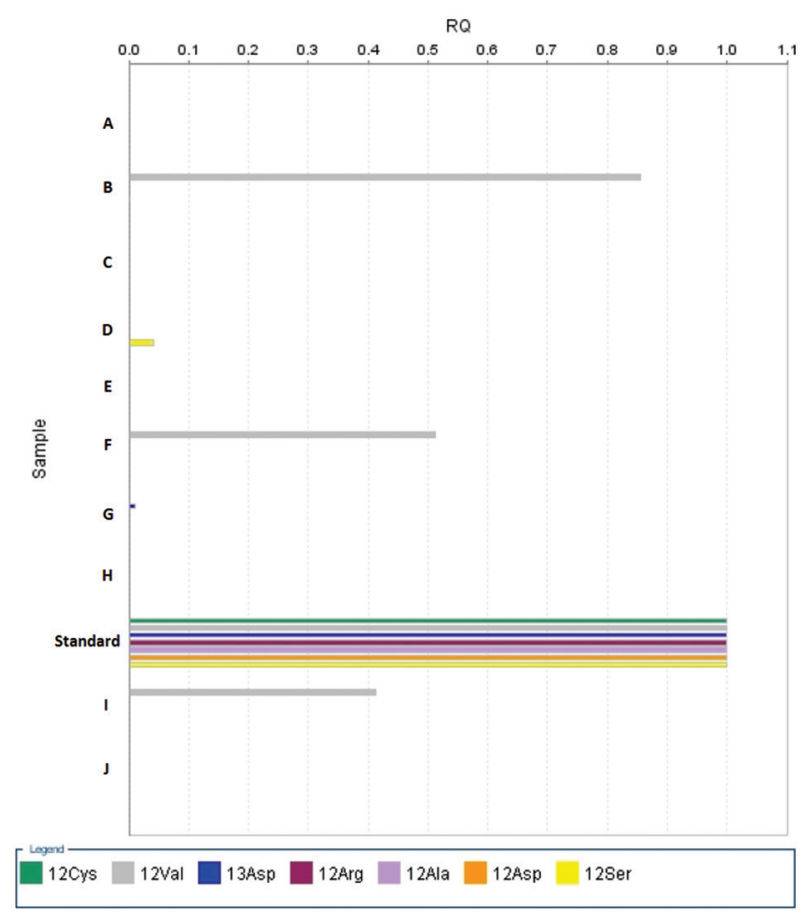

Figure 2. DxS testing assessment of KRAS mutation levels of samples 1 to 10 are shown. Samples 2, 6 and 9 contain high amounts of p.Gly12Val mutations. Sample 7 was labelled mutation positive with 1.0\% p.Gly13Asp, supporting the manufacturer's advertised sensitivity.
Table 2. Summary of Results of DxS, SNaPshot, and External Laboratory.

\begin{tabular}{|c|c|c|c|}
\hline Sample & SNaPshot & DxS & External Lab \\
\hline 1 & Wild-type & Wild-type & Wild-type \\
\hline 2 & p.Gly12Val & p.Gly12Val (85\%) & Codon 12 \\
\hline 3 & Wild-type & Wild-type & Wild-type \\
\hline 4 & $\begin{array}{l}\text { p.Gly12Ser, } \\
\text { p.Gly13Asp }\end{array}$ & p.Gly12Ser (4.1\%) & Codon 12 \\
\hline 5 & Wild-type & Wild-type & Wild-type \\
\hline 6 & p.Gly12Val & p.Gly12Val (51\%) & Gly12Val \\
\hline 7 & p.Gly13Asp & p.Gly13Asp (1.0\%) & Gly13Asp \\
\hline 8 & Wild-type & Wild-type & Wild-type \\
\hline 9 & $\begin{array}{l}\text { p.Gly12Val, } \\
\text { p.Gly13Asp }\end{array}$ & p.Gly12Val (41\%) & Codon 12 \\
\hline 10 & Wild-type & Wild-type & Wild-type \\
\hline 11 & p.Gly12Val & p.Gly12Val (32\%) & Codon 12 \\
\hline 12 & p.Gly12Asp & p.Gly12Asp (0.5\%) & Codon 12 \\
\hline 13 & Wild-type & Wild-type & Wild-type \\
\hline 14 & Wild-type & Wild-type & Wild-type \\
\hline 15 & Wild-type & Wild-type & Wild-type \\
\hline 16 & $\begin{array}{l}\text { p.Gly12Ser, } \\
\text { p.Gly12Asp, } \\
\text { p.Gly13Asp }\end{array}$ & p.Gly12Ser (1.0\%) & Codon 12 \\
\hline 17 & p.Gly13Asp & p.Gly13Asp (2.2\%) & Codon 13 \\
\hline 18 & p.Gly12Cys & p.Gly12Cys (7.6\%) & Codon 12 \\
\hline 19 & p.Gly13Asp & p.Gly13Asp (0.9\%) & $\begin{array}{l}\text { Not enough } \\
\text { DNA }\end{array}$ \\
\hline 20 & p.Gly12Ser & p.Gly12Ser (0.8\%) & Codon 12 \\
\hline 21 & p.Gly13Asp & Wild-type & Codon 12 \\
\hline 22 & Wild-type & Wild-type & Wild-type \\
\hline 23 & p.Gly12Asp & p.Gly12Asp (1.6\%) & p.Gly12Asp \\
\hline 24 & Wild-type & Wild-type & Wild-type \\
\hline 25 & p.Gly12Asp & p.Gly12Asp (1.3\%) & p.Gly12Asp \\
\hline
\end{tabular}

Results of the SNaPshot and DxS analysis that are discordant with the external laboratory are in bold italics 

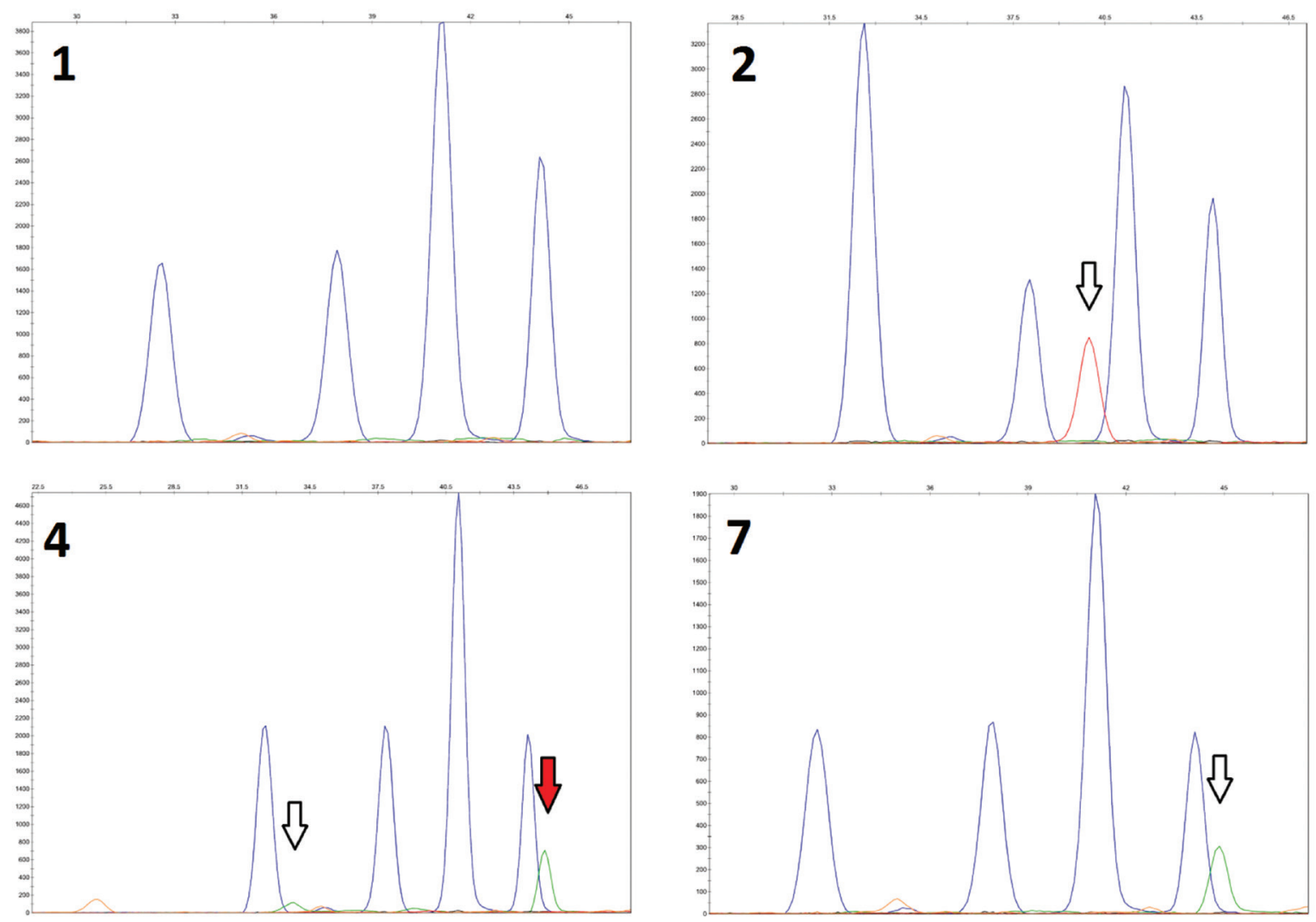

Figure 3. Illustrative results of the SNaPshot assay from samples 1, 2, 4 and 7 corresponding to the samples in Figure 2 are shown. The four large blue peaks correspond to a wild-type sequence of GGTGGC of codons 12 and 13 , as seen in sample 1. The red peak in sample 2 represents the thymine nucleotide detected in codon 12 at position 2 , causing a p.Gly $12 \mathrm{Val}$ substitution. The green peaks shown in samples 4 and 7 by the white arrows represent adenine nucleotide detection, resulting in p.Gly12Ser and p.Gly13Asp missense substitutions, respectively. The red arrow in sample D shows a p.Gly13Asp mutation detection discordant with DxS and external lab results. X-axis represents DNA fragment length and $y$-axis represents relative amount of fluorescence detected.

\section{Discussion}

Due to the long turnaround time experienced with the sending of FFPE tissue sections to an external laboratory for KRAS mutation analysis and the high cost of the Health Canada approved DxS Therascreen KRAS mutation detection kit, an alternative assay was sought out with the goal of reducing costs and turnaround time.

Methods such as RFLP or PCR with co-amplification at lower denaturation temperature (COLD-PCR) combined with High Resolution Melt (HRM) were initially considered, but ultimately deemed to be unacceptable due either to the potential need for confirmatory studies or, as in the case for HRM, the need for a large number of samples to be tested in a single run to statistically compare the melt profiles obtained. ${ }^{6,11}$ Traditional Sanger sequencing has been shown to possess a sensitivity of $\sim 20 \%, 5,12$ also falling short of DxS claim of $1 \%$ sensitivity. KRAS mutation detection using a PCR primer extension based method and fluorescently labelled ddNTPs (SNaPshot) was explored as a viable method.

After successfully demonstrating that DNA purified with the Qiagen kit produced the best amplification results, the $\mathrm{SNaPshot} \mathrm{technique} \mathrm{was} \mathrm{selected} \mathrm{for} \mathrm{comparison}$ with the DxS kit as it was reported to be as sensitive and was able to identify all mutations in codons 12 and 13 of the KRAS gene without the need for additional confirmatory assays. This technique was evaluated and found to be $95 \%$ concordant with the external lab results. It was also determined to be as sensitive as the DxS kit, both of which detected mutations below the $1 \%$ level and could be performed at a lower cost. For both assays, one sample was discordant with the external laboratory results. This could be due to limitations of the either assay or the result of tumour heterogeneity and sampling from a different location in the tumour tissue than used in the original test performed. The SNaPshot 
genotyping method also detected a p.Gly13Asp variant in four samples in addition to the correct genotype. We theorized this artefact arose as a result of the PCR amplification conditions, DNA degradation or non-specificity of primers. Further validation and troubleshooting of the $\mathrm{SNaPshot}$ method resulted in changes to the amplification conditions used and removal of the artefact. This test has been adopted for clinical use.

In New Brunswick, with an expected 50 to 100 cases per year and turnaround time of 10 days there are significant annual savings in the cost of reagents of $\$ 8,000$ - $\$ 20,000$ or more to be realized by the use of the $\mathrm{SNaPshot}$ assay instead of the DxS assay. The SNaPshot assay is versatile and can be adapted for use in any assay where point mutations must be distinguished. It has already been adapted for use in the detection of BRAF mutations in colorectal cancer ${ }^{13}$ and EGFR mutations in non-small-cell lung cancer to determine best treatment in those cancers. The SNaPshot assay is also scalable with minimal impact on cost per sample unlike the DxS kit which has a minimum batch size required to be cost effective with the reagents supplied.

With the requirement of KRAS mutation testing before administration of panitumumab, this step into personalized medicine will allow for identification of patients who are more likely to respond to anti-EGFR therapy, prevention of those with a mutation-positive KRAS gene from being exposed to the toxic effects of chemotherapy, and overall better use of health care resources.

\section{Acknowledgements}

Samuel Grummett, Tania Salesse, and Marjorie Graves are acknowledged for their technical assistance. Dr. Isabella Morava-Protzner and Dr. Tarek Rahmeh kindly provided expert advice and guidance. This study was funded by the Dalhousie Medicine Research Foundation Katelyn Robarts Summer Research Studentship.

\section{Reference}

1. Canadian Cancer Society's Steering Committee on Cancer Statistics. Canadian Cancer Statistics 2012. Toronto, ON: Canadian Cancer Society, 2012:72-8.

2. Hecht JR, Mitchell E, Neubauer MA, et al. Lack of correlation between epidermal growth factor receptor status and response to panitumumab monotherapy in metastatic colon cancer. Clin Cancer Res 2010;16:2205-2213.

3. Amado RG, Wolf M, Peeters M, Van Cutsem E, Siena S, Freeman DJ, et al. Wild-type KRAS is required for panitumumab efficacy in patients with metastatic colorectal cancer. J Clin Oncol 2008;26:1626-1634.

4. "Vectibix (panitumumab) - Letter to Health Professionals - 2008 - Health Canada." Health Canada/Santé Canada. 09
Nov. 2009. Web. 02 Oct. 2011. http://www.hc-sc.gc.ca/dhpmps/prodpharma/notices-avis/conditions/vectibix_dhcpl_ lapds_105601-eng.php

5. Soulieres D, Greer W, Magliocco AM, Huntsman D, Young S, Tsao MS, et al. KRAS testing in the treatment of metastatic colorectal cancer with anti-EGFR therapies. Current Oncology 2011;17: S31-S40.

6. Kristensen LS, Daugaard IL, Christensen M, Hamilton-Dutoit $\mathrm{S}$, Hager H, Hansen LL. Increased sensitivity of KRAS mutation detection by high-resolution melting analysis of cold-PCR products. Hum Mutat 2010;31:1366-1373.

7. Aubin F, Gill S, Burkes R, Colwell B, Kamel-Reid S, Koski S, et al. Canadian Expert Group consensus recommendations: KRAS testing in colorectal cancer. Current Oncology 2011;18: e180-e184.

8. Kamel-Reid S, Zhang T, Persons DL, Nikiforova MN, Halling KC. Validation of KRAS testing for Anti-EGFR Therapeutic Decision for Patients with Metastatic Colorectal Carcinoma. Arch Pathol Lab Med 2012;136: 26-32.

9. Van Krieken JHJM, Jung A, Kirchner T, Carneiro F, Seruca R, Bosman FT, et al. KRAS mutation testing for predicted response to Anti-EGFR therapy for colorectal carcinoma: proposal for a European quality assurance program. Virchows Arch 2008;453: 417-431.

10. Zinsky R, Bolukbas S, Bartsch H, Schirren J, Fisseler-Eckhoff A. Analysis of KRAS mutations of exon 2 codons 12 and 13 by SNaPshot analysis in comparison to common DNA sequencing. Gastroenterol Res Pract 2010:789363.

11. Do H, Krypuy M, Mitchell PL, Fox SB, Dobrovic A. High resolution melting analysis for rapid and sensitive EGFR and KRAS mutation detection in formalin fixed paraffin embedded biopsies. BMC Cancer 2008;8:142-155.

12. Vogelstein B, Kinzler K. Digital PCR. Proc Natl Acad Sci USA 1999;96:9236-9241.

13. Magnin S, Viel E, Baraquin A, Valmary-Degano S, Kantelip B, et al. A multiplex SNaPshot assay as a rapid method for detecting KRAS and BRAF mutations in advanced colorectal cancers. J Mol Diagn 2011;13: 485-492. 